\title{
Case Report \\ Complete Response of Liver Metastasis of Gastric Cancer Treated by S-1 Chemoradiotherapy: A Case Report
}

\author{
Tomonori Miyazawa, ${ }^{1}$ Kazuyu Ebe, ${ }^{2}$ Norihiko Koide, ${ }^{1}$ and Nobuhiro Fujita ${ }^{1}$ \\ ${ }^{1}$ Department of Surgery, Joetsu General Hospital, Joetsu 943-8507, Japan \\ ${ }^{2}$ Department of Radiation Therapy, Joetsu General Hospital, Joetsu 943-8507, Japan \\ Correspondence should be addressed to Tomonori Miyazawa, tmiyazaw54@yahoo.co.jp
}

Received 23 May 2012; Accepted 12 July 2012

Academic Editors: L. Beex, C. Gennatas, J. I. Mayordomo, and N. Yoshimura

Copyright (C) 2012 Tomonori Miyazawa et al. This is an open access article distributed under the Creative Commons Attribution License, which permits unrestricted use, distribution, and reproduction in any medium, provided the original work is properly cited.

\begin{abstract}
This paper presents a case of suspected liver metastasis of gastric cancer and a virtual complete response to S-1 chemoradiotherapy. A 69-year-old man underwent distal gastrectomy for gastric cancer in 2008. Multiple liver metastases occurred in 2009. He underwent 15 courses of S-1 therapy and radiation therapy (37.5 Gy). Abdominal computed tomography showed virtual complete disappearance of liver metastasis after chemoradiotherapy. Hence, this case was interpreted as a complete response. No sign of recurrence was noted 18 months after complete response was confirmed. S-1 chemoradiotherapy is likely to be effective in treating patients with liver metastases of gastric cancer.
\end{abstract}

\section{Introduction}

S-1 is an oral prodrug of fluorouracil (5-FU) with 2 biochemical modulators (gimeracil $=5$-chloro-2, 4-dihydroxypyridine inhibiting 5-FU degeneration by dihydroxypyridine dehydrogenase, and oteracil = potassium oxonate which reduces the incidence of gastrointestinal toxicity by suppressing the activation of 5-FU in the gastrointestinal tract) [1]. The SPIRITS trial showed that in metastatic gastric cancer S-1 plus cisplatin is superior to S-1 alone and, therefore, is considered as a standard treatment for advanced gastric cancer [2].

However, the use of S-1 plus cisplatin should be carefully decided in elderly patients, and if deemed inappropriate, S1 should be administered as a single agent [3]. The liver is a common site of metastasis of gastric cancer; however, the treatment for liver metastasis has not been yet established. Here, we report a case of liver metastases of gastric cancer that showed complete response (CR) to S-1 chemoradiotherapy.

\section{Case Presentation}

A 69-year-old man underwent distal gastrectomy for gastric cancer in 2008. Pathological examination showed a poorly differentiated adenocarcinoma invading the muscularis propria, without lymph node metastasis (T2a N0 M0/Stage 1B, Figures 1(a) and 1(b)). In 2009, an abdominal computed tomography (CT) scan showed multiple heterogeneous lowdensity masses in S5 and S6 of the liver (Figures 2(a)-2(b)). We diagnosed this as multiple liver metastases. The standard chemotherapy regimen for metastatic gastric cancer in Japan is S-1 plus cisplatin; however, in this case, a combination was considered inappropriate because the patient had mild renal dysfunction (creatinine clearance, $50 \mathrm{~mL} / \mathrm{min}$ ). We started S1 administration $(100 \mathrm{mg} /$ twice daily on days $1-14$, every 3 weeks) in July 2009. Abdominal CT after 5 cycles of S1 revealed a virtual complete disappearance of the tumors in S5 but not of those in S6 (Figure 2(c)-2(d)). Hepatic radiation (37.5 Gy in 15 fractions) for the tumor in S6 was performed in May 2010. Abdominal CT after radiation and 10 cycles of S-1 showed reduction in the tumor masses in S6 (Figure 2(e)).

Another series of abdominal CT performed after radiation and 15 cycles of S-1 showed complete disappearance of liver metastasis (Figure 2(f)). Hence, this case was interpreted as a CR. The patient did not experience any adverse events due to S-1 administration and irradiation. No sign of recurrence or metastasis was noted 18 months after CR was confirmed. 


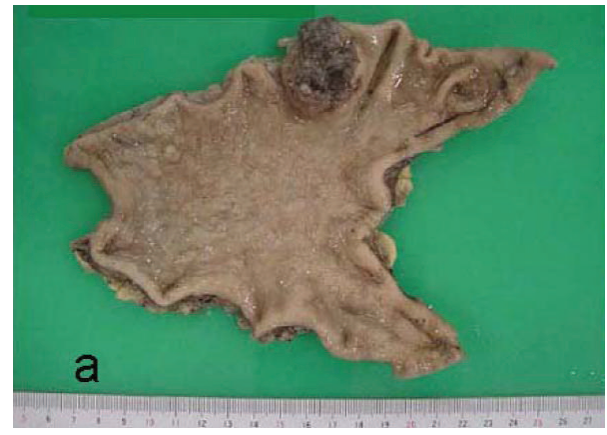

(a)

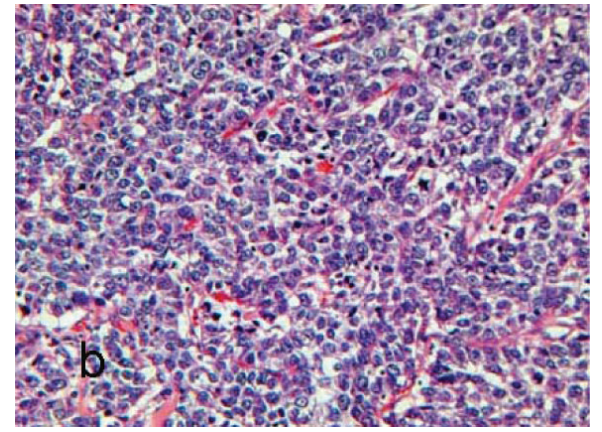

(b)

FIGURE 1: Resected specimen and pathological findings. (a) Macroscopic appearance of surgically resected specimen showing type 2 advanced gastric cancer in the corpus of the stomach. (b) Pathological examination showing a poorly differentiated adenocarcinoma (H\&E stain).

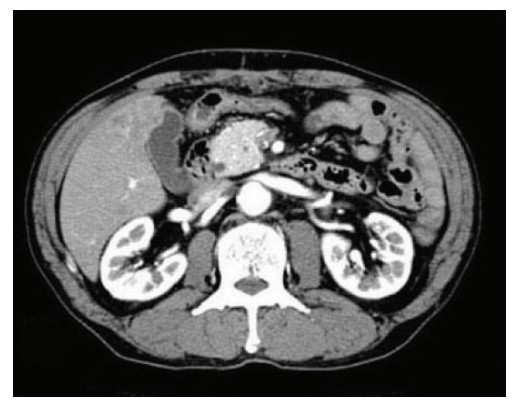

(a)

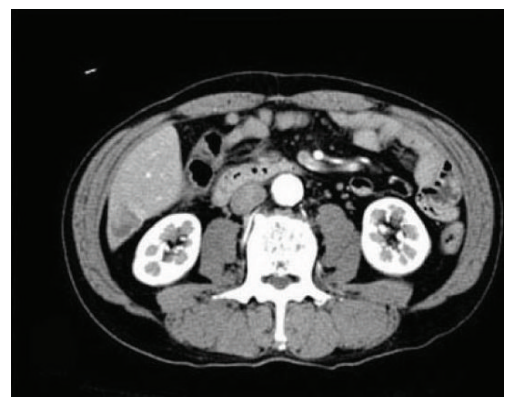

(d)

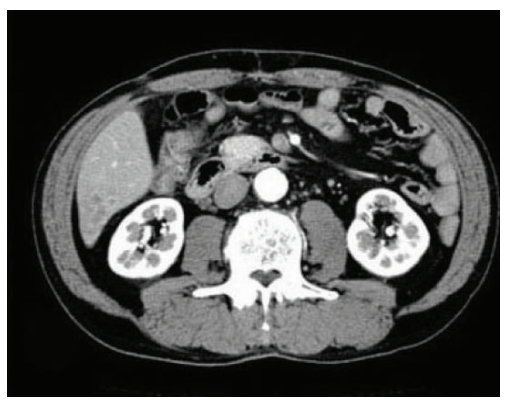

(b)

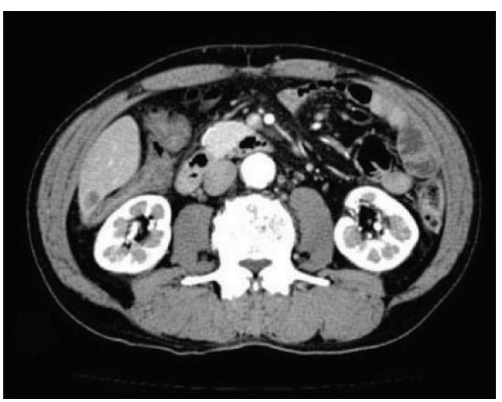

(e)

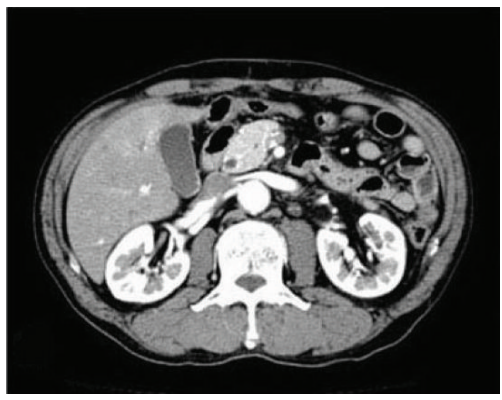

(c)

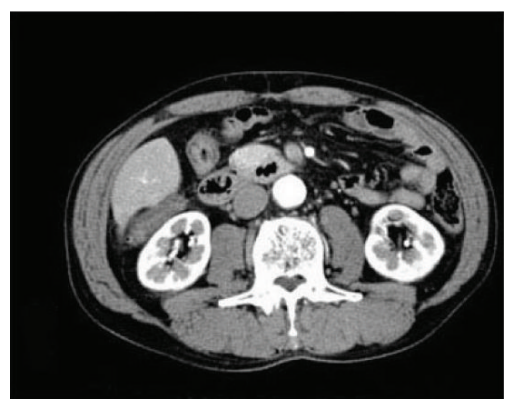

(f)

Figure 2: Abdominal computed tomography (CT) findings. (a)-(b) CT scan before chemoradiotherapy showing multiple liver metastases in S5 and S6. (c)-(d) CT scan after 5 cycles of S-1 administration showing disappearance of the tumor in S5, while tumor was still visible in S6. (e) CT scan after radiation and 10 cycles of S-1 administration showing reduction of tumor in S6. (f) CT scan after radiation and 15 cycles of S-1 administration showing disappearance of liver metastasis; hence, this case was interpreted as a complete response.

\section{Discussion}

S-1 is an oral anticancer agent containing tegafur, a metabolically activated prodrug of 5-FU, and 2 biochemical modulators [1]. S-1 is a key drug in treating gastric cancer. S1 plus cisplatin is considered a standard first-line treatment for advanced gastric cancer in Japan [2, 3]. The ACTS-GC trial demonstrated that adjuvant S-1 chemotherapy should be the standard treatment for stage II/III gastric cancer following gastrectomy with extended lymph node resection $[3,4]$. The SPIRITS trial demonstrated that S-1 plus cisplatin was superior to $\mathrm{S}-1$ alone in terms of progression-free survival (PFS) and overall survival (OS) [2]. However, subgroup analyses of the trial demonstrated that the addition of cisplatin had few benefits for elderly patients [2]. The GC0301/TOP-002 trial did not show significant superiority in the case of S-1 plus irinotecan compared with S-1 alone [5]. The JCOG 9912 trial showed cisplatin plus irinotecan was not superior to S-1 or continuous infusion of 5FU, and that S-1 was noninferior to 5-FU [6]. Therefore, for convenience, oral administration of S-1 could replace intravenous 5-Fu in the treatment of advanced gastric cancer and could be considered a standard first-line treatment [6].

S-1 is usually administrated for 4 weeks, followed by a 2-week drug-free period. Adverse reactions related to S-1 therapy commonly begin to appear 2-3 weeks after treatment 
starts [7]. The 2-week regimen of S-1 followed by a 1week drug-free period might mitigate adverse reactions and prolonged medication period [7]. Two phase II studies of the 2-week regimen of S-1 showed equivalent OS and PFS compared with other conventional chemotherapeutic regimens $[8,9]$. Our patient did not experience any adverse events during the 15 cycles of the 2 -week regimen of S- 1 .

The liver is a common site of metastasis of gastric cancer; however, the treatment for liver metastasis of gastric cancer has not been well established. The results of metastectomy for liver metastasis of gastric cancer have been disappointing; thus, metastectomy of the liver should be performed in selected patients as part of multidisciplinary treatments [10, 11]. Local-regional radiation plus systemic chemotherapy administered as postoperative treatment was effective for controlling recurrence of gastric cancer [12]. In our case, hepatic radiation was efficacious against liver metastasis. Nakamura reported on the efficacy of hepatic radiation plus systemic chemotherapy, including S-1, for liver metastasis of gastric cancer [13]. Addition of the radiation as salvage might be useful for the patients with liver metastasis of gastric cancer.

In conclusion, we reported a case of suspected liver metastasis that showed CR to S-1 chemoradiotherapy. Thus, S-1 chemoradiotherapy is likely to be effective in treating patients with liver metastasis of gastric cancer. S-1 has recently been approved by the EMA, product name Teysuno.

\section{Conflict of Interests}

The authors have no conflict of interests to declare.

\section{References}

[1] T. Shirasaka, "Development history and concept of an oral anticancer agent S-1 (TS-1): its clinical usefulness and future vistas," Japanese Journal of Clinical Oncology, vol. 39, no. 1, pp. 2-15, 2009.

[2] W. Koizumi, H. Narahara, T. Hara et al., "S-1 plus cisplatin versus S-1 alone for first-line treatment of advanced gastric cancer (SPIRITS trial): a phase III trial," The Lancet Oncology, vol. 9, no. 3, pp. 215-221, 2008.

[3] T. Sano and Y. Kodera, "Japanese gastric cancer treatment guidelines 2010 (ver. 3)," Gastric Cancer, vol. 14, no. 2, pp. 113-123, 2011.

[4] S. Sakuramoto, M. Sasako, T. Yamaguchi et al., "Adjuvant chemotherapy for gastric cancer with S-1, an oral fluoropyrimidine," New England Journal of Medicine, vol. 357, no. 18, pp. 1810-1820, 2007.

[5] H. Narahara, H. Iishi, H. Imamura et al., "Randomized phase III study comparing the efficacy and safety of irinotecan plus S-1 with S-1 alone as first-line treatment for advanced gastric cancer (study GC0301/TOP-002)," Gastric Cancer, vol. 14, no. 1, pp. 72-80, 2011.

[6] N. Boku, S. Yamamoto, H. Fukuda et al., "Fluorouracil versus combination of irinotecan plus cisplatin versus S-1 in metastatic gastric cancer: a randomised phase 3 study," The Lancet Oncology, vol. 10, no. 11, pp. 1063-1069, 2009.

[7] Y. Kimura, N. Kikkawa, S. Iijima et al., "A new regimen for S-1 therapy aiming at adverse reaction mitigation and prolonged medication by introducing a 1-week drug-free interval after each 2-week dosing session: efficacy and feasibility in clinical practice," Gastric Cancer, vol. 6, no. 1, pp. 34-39, 2003.

[8] H. C. Jeung, S. Y. Rha, S. J. Shin et al., "A phase II study of S1 monotherapy administered for 2 weeks of a 3-week cycle in advanced gastric cancer patients with poor performance status," British Journal of Cancer, vol. 97, no. 4, pp. 458-463, 2007.

[9] J. H. Lim, M. H. Lee, H. G. Kim et al., "Three-Weekly S-1 monotherapy as first-line treatment in elderly patients with recurrent or metastatic gastric cancer," Gut and Liver, vol. 4, no. 4, pp. 503-507, 2010.

[10] K. Shirabe, S. Wakiyama, T. Gion et al., "Hepatic resection for the treatment of liver metastases in gastric carcinoma: review of the literature," HPB, vol. 8, no. 2, pp. 89-92, 2006.

[11] S. P. Kerkar, C. D. Kemp, and I. Avital, "Liver resections in metastatic gastric cancer," HPB, vol. 12, no. 9, pp. 589-596, 2010.

[12] J. S. Macdonald, S. R. Smalley, J. Benedetti et al., "Chemoradiotherapy after surgery compared with surgery alone for adenocarcinoma of the stomach or gastroesophageal junction," New England Journal of Medicine, vol. 345, no. 10, pp. 725730, 2001.

[13] R. Nakamura, Y. Saikawa, K. Kumagai et al., "A patient with gastric cancer and liver metastases successfully treated with combination chemotherapy including S-1," International Journal of Clinical Oncology, vol. 12, no. 4, pp. 295-299, 2007. 


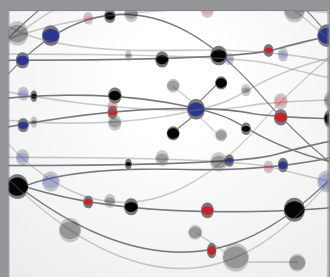

The Scientific World Journal
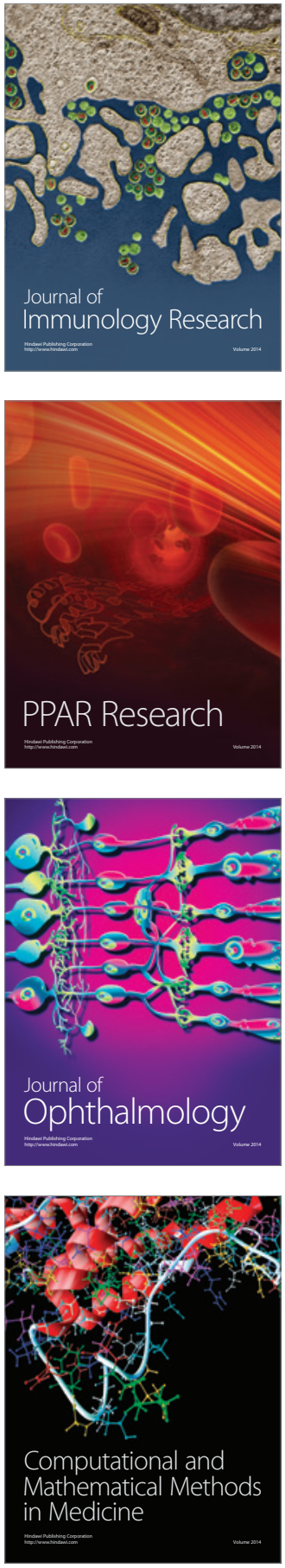

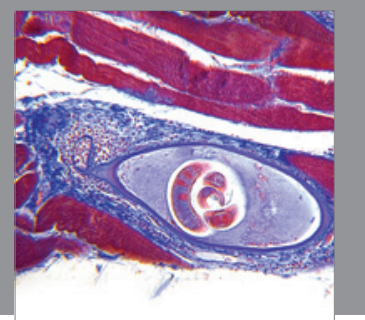

Gastroenterology

Research and Practice
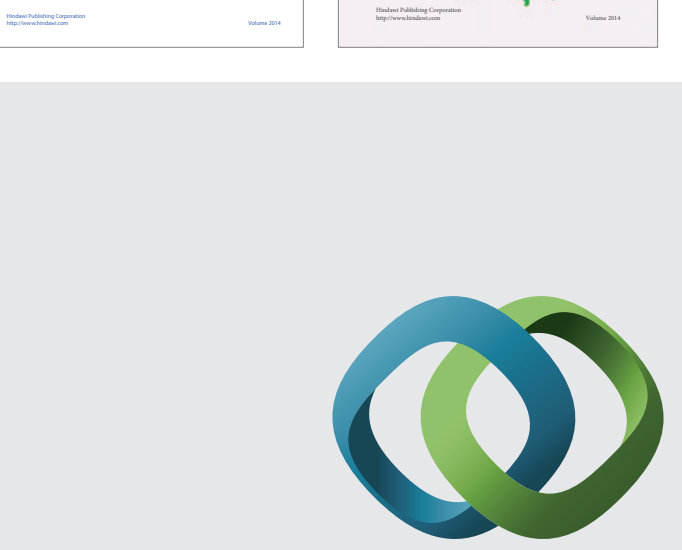

\section{Hindawi}

Submit your manuscripts at

http://www.hindawi.com
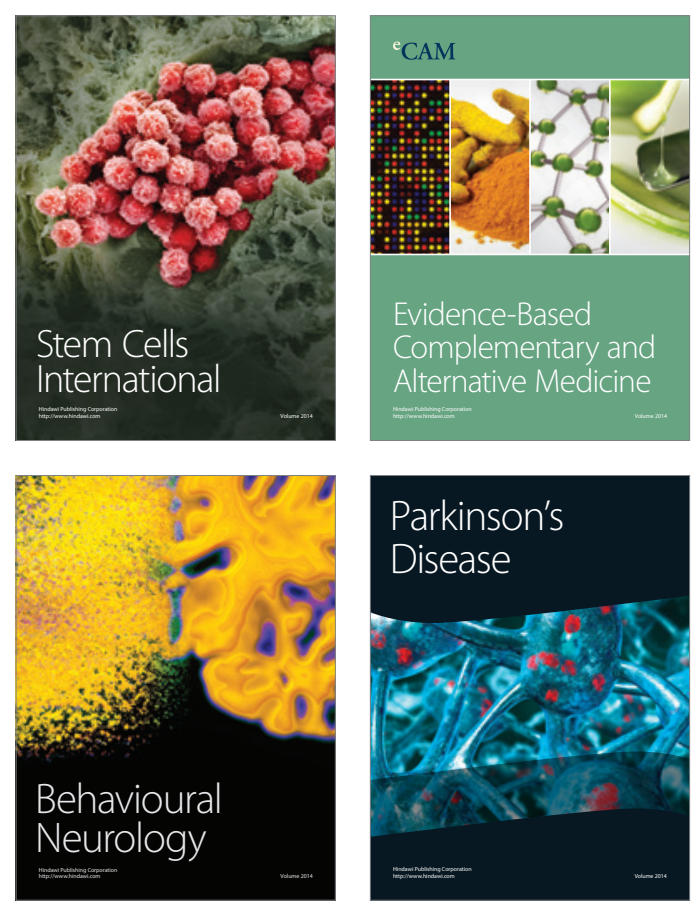

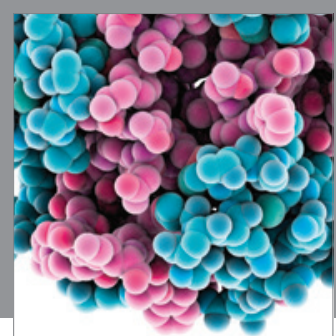

Journal of
Diabetes Research

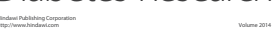

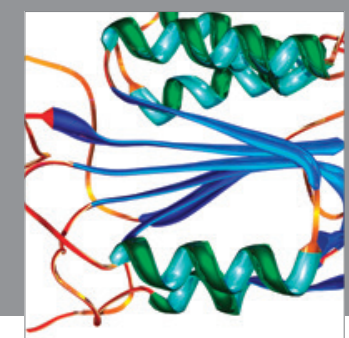

Disease Markers
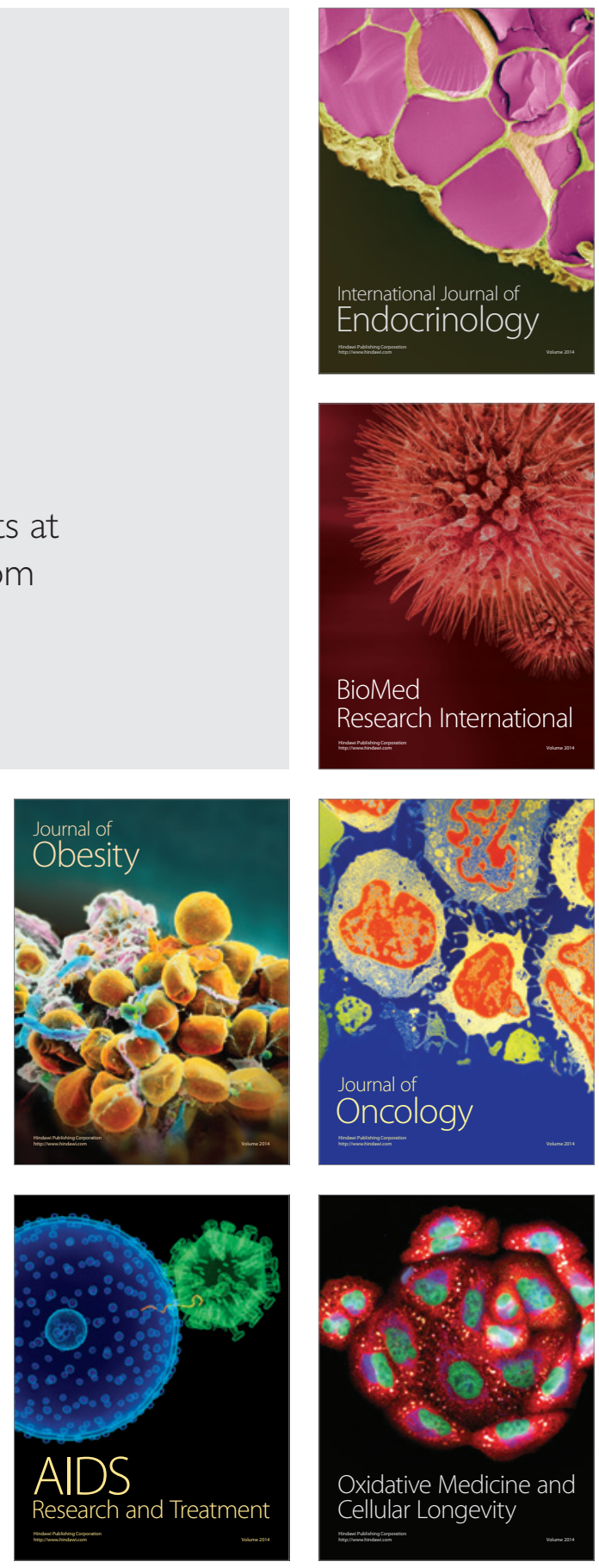